\title{
Minimization of Threshold-Current Dependence of Quantum Dot Laser Using InN
}

\author{
M. A. Humayun ${ }^{1}$, M. A. Rashid ${ }^{2}$, M. Abdul Malek ${ }^{3}$ \\ ${ }^{1}$ Dept. of Electrical and Electronic Engineering, Eastern University, Dhaka-1205, Bangladesh \\ ${ }^{2}$ Dept. of EEE, Noakhali Science \& Technology University, Noakhali 3814, Bangladesh. \\ ${ }^{3}$ Faculty of Engineering and Information Sciences, University of Wollongong in Dubai, Dubai, UAE.
}

\begin{tabular}{l} 
Article Info \\
\hline Article history: \\
Received Sep 1, 2018 \\
Revised Mar 31, 2019 \\
Accepted May 29, 2019 \\
\hline
\end{tabular}

\section{Keyword:}

Internal loss

Mirror loss

Modal gain

Turn-on delay

\begin{abstract}
This paper focuses on the effect of threshold current density on different characteristics of quantum dot-based laser. To investigate the effect, different characteristics have been analyzed using quantum dot as the active layer material of the laser structure. Performance improvement of quantum dot laser using $\mathrm{InN}$ has been achieved in terms of minimization of the threshold current dependence such as mirror loss, modal gain, turn-on delay and so forth. Numerical results have been analyzed considering the values of threshold current densities of GaN, AlN and InN based quantum dot lasers. Analytical results show that internal loss increases linearly with the increase of the threshold current density. However, other characteristics like mirror loss, modal gain, turn-on delay have nonlinear dependence on the threshold current density for any material used in the active layer. The threshold current density of InN quantum dot-based laser is lower than that of other existing quantum dot-based lasers. Analytical results ascertained that mirror loss has been increased a little bit. Conversely, internal loss and modal gain have been minimized considerably using InN based quantum dot in the active layer of the laser structure. In addition, turn-on delay time has been also minimized significantly.
\end{abstract}

Copyright (C) 2019 Institute of Advanced Engineering and Science. All rights reserved.

\section{Corresponding Author:}

M. A. Rashid,

Department of EEE, Noakhali 3814, Bangladesh.,

Noakhali Science \& Technology University,

Sonapur, University Rd, 3814, Bangladesh.

Email: rashid68@yahoo.com

\section{INTRODUCTION}

Semiconductor laser has been considered as one of the most vital devices in the field of optoelectronic semiconductor technology over the decades [1]. In early days homostructured semiconductor lasers were fabricated using bulk semiconductor materials. These homostructured devices suffered from high threshold current density to create lasing action. In addition to that these devices were only able to operate at cryogenic temperature. The active region of a bulk semiconductor laser was large enough to absorb light with a plethora of atoms. Therefore, it was necessary to pump in a lot of electrons and holes to overcome the photonic reabsorption and generate light in a self-sustaining way. More requirement of current means greater power requirement and more performance problems due to heat caused by high power source used for the lasing action to take place. On the other hand, a wide active region also causes a greater number of uncontrolled spontaneous emissions of photons. As a result of the uncoltrolled emission of photons optical noise is generated. Another major problem of large active region was a large linewidth enhancement factor (LEF). Due to the increase in threshold current and modal and material gains, the index of refraction fluctuated as well. All these variations affected the uniformity of the wavelength of the standing waves inside the laser cavity [2]. Therefore reduction of threshold current is one of the major issues nowadays. 
Advanced performance of heterostructure lasers compared to homostructured lasers made them capable for using in optical communication systems since 1978. However, bulk materials in the active region blocked further improvements of heterostructure lasers and led to the development of quantum well lasers motivated by the strong modification of properties of a semiconductor crystal in low dimensional heterostructures. These limitations of bulk lasers have been minmized by introducing quantum well in the active layer of the laser structure in steade of the conventional bulk semiconductor materials. Nevertheless it is also reveal that quantum well lasers are still suffering from few other performance problems [3] such as: non-equilibrium carrier spreading out of the cavity, facet overheating due to surface recombination,non-radiative recombination enhanced by dislocation growth due to carrier diffusion to dislocations, no possibility of free-standing micro stripes or micro cavities and large ground state population time associated with QWLs restricted the achievement of better performances of the laser.

Further enhancement of device characteristics is expected for lasers with lower dimensionality of the active region, such as quantum wire and especially quantum dot. In quantum dot, the motion of carriers is restricted from all the three directions. It has been reported by different gropups of researchers that suppression of temperature dependence of threshold current and some other problems of quantum well lasers has been reduced significently by using quantum dot as the active layer material of the laser structure [4]-[5].

In 2000 it has been reported by Bimberg and his group that since the innovation of lasing in self-assembled quantum dots, this material has gained increasing significance to the researchers due to their potentiality in the optoelectronic device applications [3]. Recently group III-V nitride materials have become the subject of escalating importance to the researchers for their latent significance in improving the device characteristics [6]. The group-III nitrides are especially used to fabricate UV, green and blue LED and LASERs as well as PV cells due to their wide range of frequency spectrum [7]. The effect of different parameters like temperature, lattice constant, photon life time, optical feedback, operating time, cavity length on the different characteristics of the quantum dot based laser have been investigated comprehensively in the last few years [8]-[12]. Additionally, inhomogeneous line broadening of quantum dot based laser has also been studied extensively in previous years [10], [13]-[14]. From the outcome of the few recent studies it has been revealed that InN quantum dot plays a significant role on the laser charecteriatics [8]-[9],[15]. However, the effects of threshold current density on the laser characteristics is one of the least studied feature. Therefore, our recent research has been devoted to analyze the effect of threshold current on the laser dynamics.

Therefore, in this paper, we have investigated rigorously the effect of threshold current density on the loss and gain mechanism along with the modulation response of quantum dot based laser. The loss mechanism includes internal loss and mirror loss. We have also investigated the modal gain phenomena among the different types of gains of quantum dot based laser. Additionally, among the characteristics related to modulation phenomena such as turn-on delay time and the relaxation oscillation frequency have also been demonstrated. These important characteristics have been analyzed through simulation and mathematical approach using MATLAB. After that the investigations were carried out using the values of threshold current densities obtained for AlN, $\mathrm{GaN}$, InN based quantum dot laser. Earlier studies reveal that the threshold current densities of AlN and GaN based quantum dot based lasers are $13 \mathrm{Acm}-2$ and $12 \mathrm{Acm}-2$ respectively. It has been reported that the threshold current density of InN quantum dot laser is $10 \mathrm{Acm}-2$ [16].

This paper highlights the significant minimization of threshold current dependence of mirror loss and internal loss, required modal gain to overcome these losses using $\mathrm{InN}$ based quantum dot as the active layer material of the laser structure. This paper also reports that the high speed operation of laser along with the enhanced relaxation oscillation frequency has been achieved using $\mathrm{InN}$ based quantum dot in the active layer of the laser structure.

\section{RESEARCH METHOD}

This section presents the materials and methods of analyzing the effect of threshold current density on the loss, gain, turn-on delay and modulation response of quantum dot based laser using InN based quantum dot in the active layer of the laser structure. Sub-section 2.1 presents the detail of the device structure. Subsection 2.2 presents the Threshold current dependence of losses and gain characteristics of quantum dot based laser. Among the loss characteristics, the internal loss and the mirror loss have been considered in this research work. Sub-section 2.3 presents the threshold current dependence of turn-on delay and modulation response of quantum dot based laser. 


\subsection{Layer Structure of Quantum Dot Based Laser}

Figure 1 presents the layer structure of the quantum dot based laser used in this research work. Schematic structure of InN based quantum dot based laser consists of c-plane sapphire (oriented along 0001 direction) wafer as the substrate along with $77 \mathrm{~nm}$-thick $\mathrm{n}+\mathrm{GaN}$ contact layer; a $1000 \mathrm{~nm}$ thick $\mathrm{Al}_{0.13} \mathrm{Ga}_{0.87} \mathrm{~N}$ lower cladding layer; a $117 \mathrm{~nm}$-thick $\mathrm{n}$ - $\mathrm{In}_{0.82} \mathrm{Ga}_{0.18} \mathrm{~N}$ guiding layer; a $2.7 \mathrm{~nm}$-thick active region with single layer undoped InN QDs; a 117 nm-thick p $\mathrm{In}_{0.82} \mathrm{Ga}_{0.18} \mathrm{~N}$ guiding layer; a $1000 \mathrm{~nm}$ thick p- $\mathrm{Al}_{0.13} \mathrm{Ga}_{0.87} \mathrm{~N}$ upper cladding layer; $77 \mathrm{~nm}$-thick $\mathrm{p}+\mathrm{GaN}$ contact layer. Thus optical confinement layer thickness is $236.7 \mathrm{~nm}$.

\begin{tabular}{|c|}
\hline Contact layer p+ GaN $77 \mathrm{~nm}$ \\
\hline Upper cladding layer $\mathrm{p}$ Al0.13Ga0.87N $1000 \mathrm{~nm}$ \\
\hline Guiding layer $\mathrm{p}$ In0.82Ga0.18N $117 \mathrm{~nm}$ \\
\hline Active region InN undoped QDs $2.7 \mathrm{~nm}$ \\
\hline Guiding layer n In0.82Ga0.18N $117 \mathrm{~nm}$ \\
\hline Lower cladding layer n Al0.13Ga0.87N $1000 \mathrm{~nm}$ \\
\hline Contact layer n+ GaN 77 nm \\
\hline Substrate c- sapphire(0001) \\
\hline
\end{tabular}

Figure 1. Schematic diagram of quantum dot based laser using InN.

\subsection{Losses and Gain}

Threshold current density is one of the most important parameters of a laser that affects the overall performance of the lasing action. The threshold current density has significant effect on the internal loss [9], [12]. The internal loss is directly related to the threshold current density. The relationship between internal loss and the threshold current density is described in [17] which is given by the following equation

$$
\alpha_{i}=\alpha_{i o}+k_{a} J_{t h}
$$

Here, $\quad \alpha_{i}$ is the initial loss of quantum dot laser,

$\alpha_{i 0}$ is the initial loss component related to any other loss mechanism like mode lock and scattering,

$k_{a}$ is the parameter related to the geometry of the laser waveguide,

$J_{t h}$ is the threshold current density.

From equation (1) it is ascertained that the internal loss increases linearly with the increase of threshold current density. As a result, higher mirror loss occurs at higher threshold current density. So, it is anticipated that InN based quantum dot in the active layer of the laser structure would offer the lowest internal loss among the three materials used in this study to investigate this phenomenon.

Now we will calculate the mirror loss as a function of threshold current density. Mirror loss is not a direct function of threshold current density but it is a function of maximum saturated optical gain. The relationship between the gain and the maximum mirror loss is given by the following equation

$\alpha_{m}^{\max }=g^{\text {sat }}-\alpha_{i}$

Where $g^{\text {sat }}$ is the saturation optical gain.

Again, the saturation gain $g^{\text {sat }}$ is related to the gain and the transparency current density by the following equation

$$
g=g^{s a t}\left(\frac{J-J_{0}}{J_{0}}\right)
$$

Where $J$ is the radiative recombination within the quantum dot in ground state and $J_{0}$ is the transparency current density [17]. 
On the other hand, the gain is a direct function of threshold current density [16]. The relationship between threshold current density and modal gain is given by the following equation

$$
g=g_{0} \ln \left(\frac{J_{t h}}{J_{0}}\right)
$$

Where $g_{0}$ is the gain coefficient and is the threshold current density.

Now the mirror loss has been calculated as a function of threshold current density using equation (1)(4). This relationship is deduced as follows

$$
\alpha_{m}^{\max }=\frac{J_{0} g_{0}}{J-J_{0}} \ln \left(\frac{J_{t h}}{J_{0}}\right)-\alpha_{i o}-k_{a} J_{t h}
$$

\subsection{Turn-on Delay and Modulation Response}

Among the output characteristics turn-on delay and modulation response have threshold current dependence [18]. Therefore, we have also considered these characteristics of laser to investigate extensively. When a bias current is applied to the laser generally a few nano seconds of delay occurs before the stimulated emission [19]. On the other hand, the stimulated emission occurs beyond the threshold value of the biasing current i.e. threshold current. So, the turn-on delay is directly dependent on the value of threshold current. The relationship between the threshold current density and the turn-on delay time is given by the following equation

$$
t_{d}=\tau \ln \left(\frac{J}{J-J_{t h}}\right)
$$

Where ${ }^{t_{d}}$ is the turn-on delay time and $\tau$ is the carrier life-time.

Now we will investigate the threshold current dependence of the relaxation oscillation frequency of quantum dot based laser. The relationship of the relaxation oscillation frequency with the threshold current density is given by the following relationship:

$$
f_{r}=\frac{1}{2 \pi} \sqrt{\frac{1}{\tau \tau_{p h}}\left(\frac{J_{0}}{J_{t h}}-1\right)}
$$

Where $f_{r}$ is the relaxation oscillation frequency and $\tau_{p h}$ is the photon life-time.

\section{RESULTS AND ANALYSIS}

The threshold current dependence of different characteristics of laser has been investigated extensively in this research work using MATLAB. The effect of threshold current density on different types of losses, gain and modulation characteristics of quantum dot based laser has been studied interms of internal loss, mirror loss and the modal gain. After that these characteristics has been analyzed using the three different materials namely InN, GaN and AlN based quantum dot in the active layer of the device structure. The considering the corrosponding values of the threshold current densities. The outcone of the numarical analysis are described below.

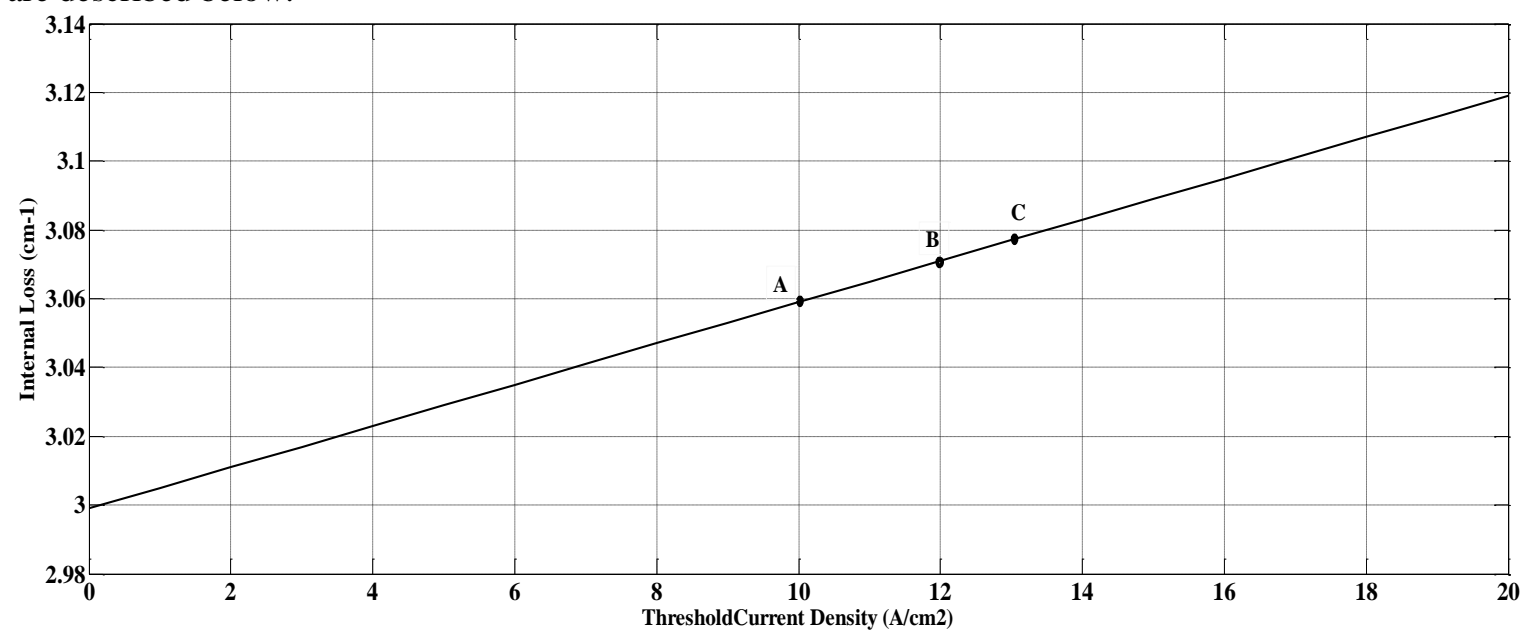

Figure 2. Effect of threshold current density on internal loss of quantum dot lasers using InN (A), GaN (B) and $\mathrm{AlN}(\mathrm{C})$ based quantum dot as the active layer material of the laser structure. 
Figure 2 shows the effect of the threshold current density on the internal loss of quantum dot based laser. From the figure it is ascertained that the internal loss increases linearly with the increase of threshold current density. The points on the curve, A, B and C indicate the values of internal loss using $\mathrm{InN}, \mathrm{GaN}$ and AlN based quantum dot in the active layer of the laser structure respectively. The values of internal loss obtained for InN, GaN and AlN based quantum dot lasers are $3.06 \mathrm{~cm}^{-1}, 3.072 \mathrm{~cm}^{-1}$ and $3.08 \mathrm{~cm}^{-1}$ respectively. Therefore, the analysis of threshold current dependence of the absorption loss ascertains that the InN based quantum dot in the active layer provide the lowest intrernal loss among the group-III nitride trios considered in this research work. The internal loss of quantum dot based laser has been reduced $0.39 \%$ and $0.65 \%$ using $\mathrm{InN}$ with respect to those of using $\mathrm{GaN}$ and $\mathrm{AlN}$ respectively.

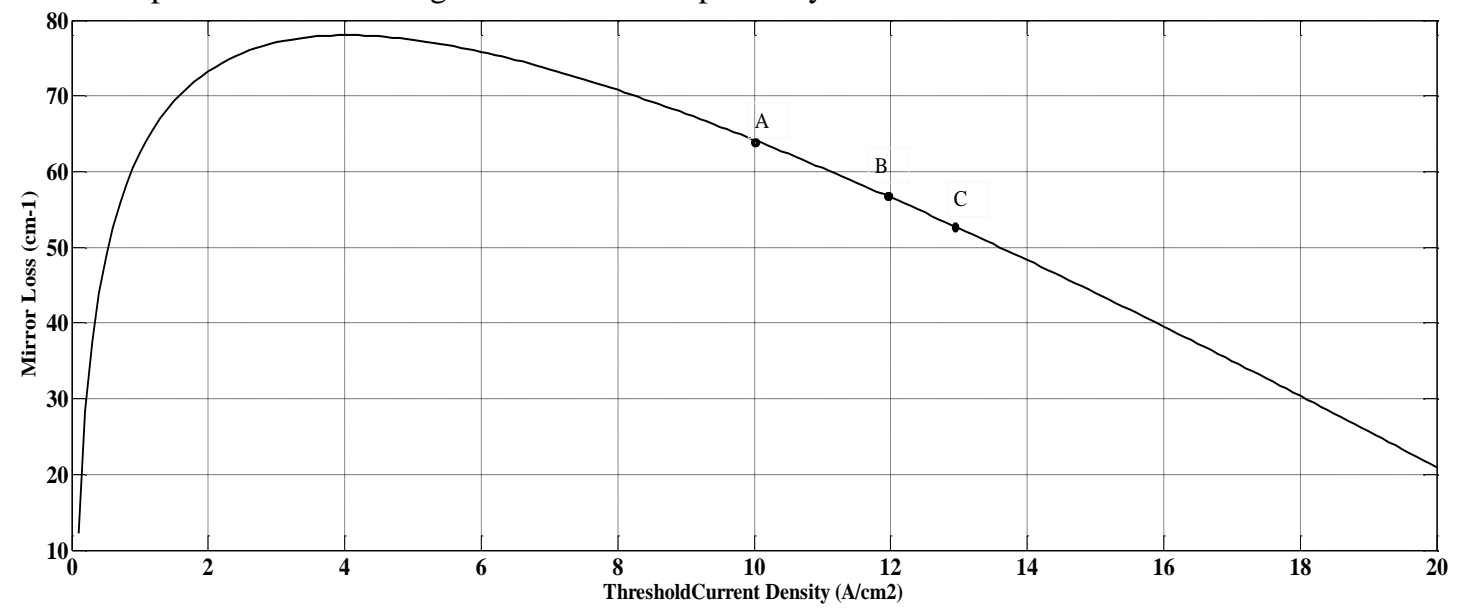

Figure 3. Effect of threshold current density on mirror loss of quantum dot lasers using InN (A), GaN (B) and AlN (C) based quantum dot as the active layer material of the laser structure.

Figure 3 shows dependence of mirror loss of quantum dot laser on the threshold current density. The mirror loss increases initially with the increase of threshold current density. For the value of threshold current density of $4 \mathrm{Acm}^{-2}$ the mirror loss of quantum dot laser reaches to the maximum point and starts to decrease slowly afterwards. The points on the curve A, B and C indicate the values of mirror loss using InN, GaN and AlN based quantum dot in the active layer of the laser structure. The values of internal loss obtained from the figure for InN, GaN and AlN based quantum dot lasers are $63 \mathrm{~cm}^{-1}, 59.7 \mathrm{~cm}^{-1}$ and $57 \mathrm{~cm}^{-1}$ respectively. The analysis of threshold current dependence of the mirror loss shows that the InN based quantum dot in the active layer provides $5.23 \%$ and $9.25 \%$ higher mirror loss than that of using GaN and AlN respectively although it offers the lowest value for the internal loss, which affects the total loss significently.

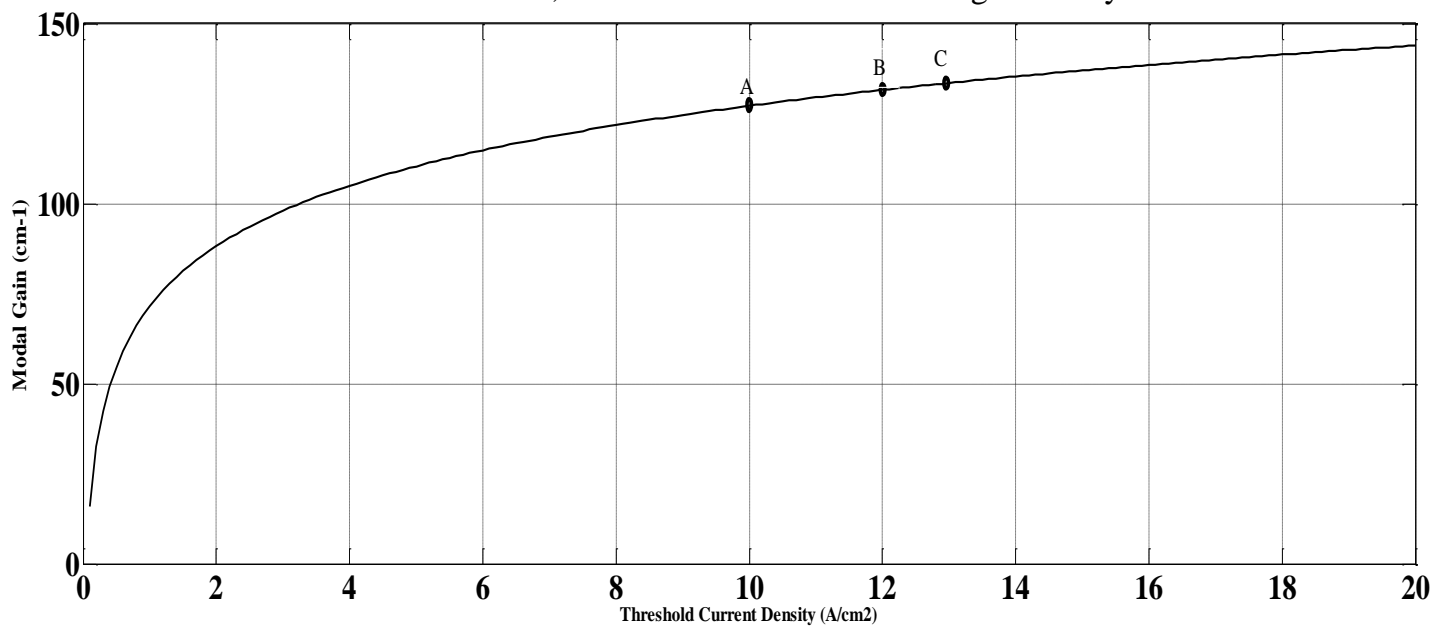

Figure 4. Effect of threshold current density on modal gain of quantum dot lasers using InN (A), GaN (B) and AlN (C) based quantum dot as the active layer material of the laser structure.

Figure 4 shows the effect of threshold current density on the modal gain characteristics of quantum dot based laser. The modal gain is the gain required to over come the total losses. Hence, lower value of modal gain is necessery to improve the performance of the laser [13]. The modal gain of the laser increases nonlinearly with the increase of threshold current density as shown in the above figure. The points on the curve A, B and 
$\mathrm{C}$ indicates the values of modal gain of $\mathrm{InN}, \mathrm{GaN}$ and $\mathrm{AlN}$ based quantum dot lasers. The values of modal gain obtained from the figure for InN, GaN and AlN quantum dot based lasers are $125 \mathrm{~cm}^{-1}, 135 \mathrm{~cm}^{-1}$ and $137 \mathrm{~cm}^{-}$ ${ }^{1}$ respectively. Therefore, the analysis of threshold current dependence of the modal gain ascertains that the InN based quantum dot in the active layer offers $8 \%$ and $9.6 \%$ lower modal gain required to overcome the total loss.using InN with respect to those of using GaN and AlN respectively.

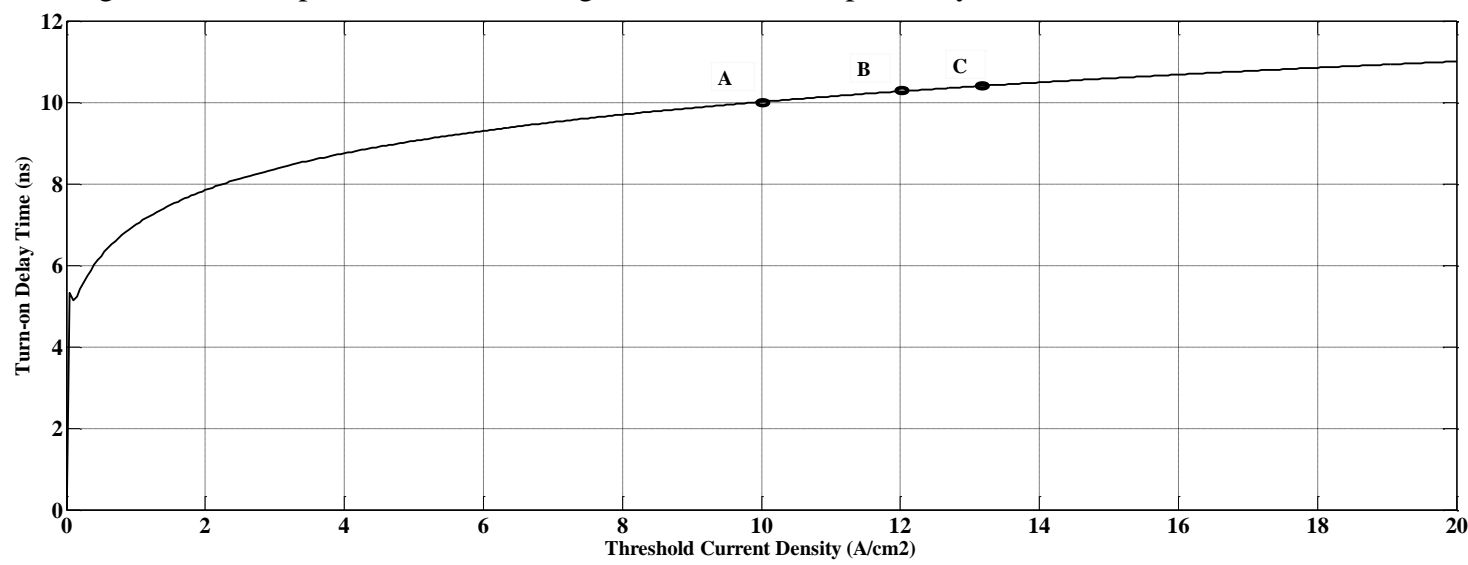

Figure 5. Effect of threshold current density on turn-on delay time of quantum dot lasers using $\operatorname{InN}(\mathrm{A}), \mathrm{GaN}$ (B) and AlN (C) based quantum dot as the active layer material of the laser structure.

Figure 5 represents the effect of threshold current density on the turn-on delay characteristics of quantum dot based laser. As shown in the above figure, the turn on delay of the laser increases nonlinearly with the increase of threshold current density. The points on the curve A, B and C indicate the values of turn-on delay times of $\mathrm{InN}, \mathrm{GaN}$ and AlN quantum dot based lasers respectively. The values of turn-on delay time of the InN, GaN and AlN quantum dot based lasers are $9.9 \mathrm{nS}, 10.25 \mathrm{nS}$ and $10.37 \mathrm{nS}$ respectively. Therefore, the analysis of threshold current dependence of the turn-on delay time ascertains that the InN based quantum dot in the active layer provides $3.53 \%$ and $4.75 \%$ lower mirror loss than that of using GaN and AlN respectively, which assures that the higher modulation speed has been acheived by using InN based quantum dot in the active layer of the laser structure than the other materials used in this research work.

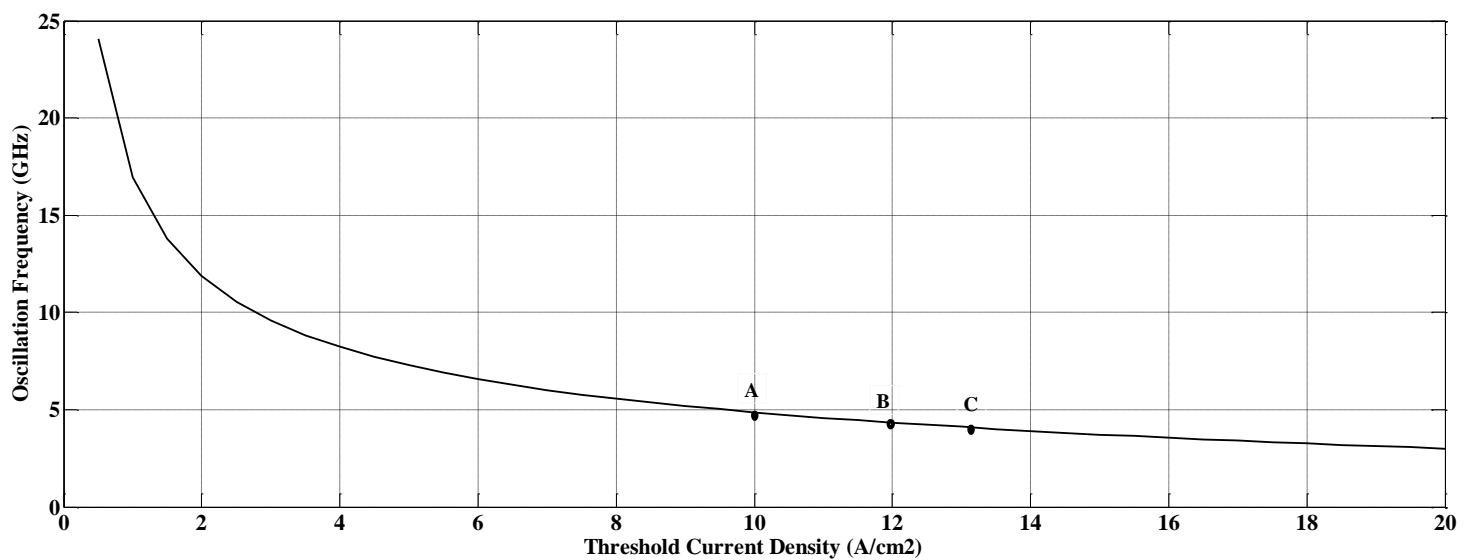

Figure 6. Effect of threshold current density on laser oscilation frequency of quantum dot lasers using InN (A), GaN (B) and AIN (C) based quantum dot as the active layer material of the laser structure.

Figure 6 represents the effect of threshold current density on the oscillation frequency of quantum dot based laser. From the figure, it is revealed that the oscillation frequency decrease non-uniformly with the increase of threshold current density. The points on the curve A, B and C indicates the values of laser oscillation frequency using $\mathrm{InN}, \mathrm{GaN}$ and $\mathrm{AlN}$ based quantum dot in the active layer of the laser structure.The oscillation frequency of the laser obtained by using $\mathrm{InN}, \mathrm{GaN}$ and $\mathrm{AlN}$ are $4.86 \mathrm{GHz}, 4.33 \mathrm{GHz}$ and $4.12 \mathrm{GHz}$ respectively. Therefore, the analysis of threshold current dependence of the frequency response reveales that the InN based quantum dot in the active layer provide $10.91 \%$ and $17.28 \%$ higher laser oscillation frequency, which enables the high speed data transmission with lower noise through optical networks. 


\section{DISCUSSION}

This section presents the sumary of the findings of this research work in a tabular form. Table 1 prersents the comparision of internal loss, mirror loss and the total loss, turn-on delay and oscilation frequency of a quantum dot based laser using InN, GaN and AlN .

Table 1. The comparision of threshold-current dependence of quantum dot laser parameters

\begin{tabular}{cccccc}
\hline $\begin{array}{c}\text { Active layer } \\
\text { Material }\end{array}$ & $\begin{array}{c}\text { Internal Loss } \alpha_{\mathrm{i}} \\
\left(\mathrm{cm}^{-1}\right)\end{array}$ & $\begin{array}{c}\text { Mirror Loss } \alpha_{\mathrm{m}} \\
\left(\mathrm{cm}^{-1}\right)\end{array}$ & $\begin{array}{c}\text { Modal Gain } \\
\left(\mathrm{cm}^{-1}\right)\end{array}$ & $\begin{array}{c}\text { Turn-on delay } \\
(\mathrm{nS})\end{array}$ & $\begin{array}{c}\text { Oscilation } \\
\text { frequency }(\mathrm{GHz})\end{array}$ \\
\hline $\mathrm{InN}$ & 3.06 & 63 & 125 & 9.9 & 4.86 \\
$\mathrm{GaN}$ & 3.072 & 59.7 & 135 & 10.25 & 4.33 \\
$\mathrm{AlN}$ & 3.08 & 57 & 137 & 10.37 & 4.02 \\
\hline
\end{tabular}

From the Table 1 it is clear that mirror loss and the oscilation frequency have been increased a little bit. However, the internal loss and modal gain required to overcome the total loss and the turn-on delay time have been minimized considerably using InN based quantum dot in the active layer of the laser structure.

\section{CONCLUSION}

In this paper we have analyzed the effect of threshold current density on major loss and gain phenomena along with the modulation characteristics of quantum dot based laser. These important phenomena were investigated extensively in terms of internal loss, mirror loss and modal gain and turn-on delay time of the laser. From the results it is revealed that the internal loss has been minimized by $0.39 \%$ and $0.65 \%$ using InN based quantum dot in the active layer of the laser structure with respect to that of using GaN and AlN respsctively. Additionally, the modal gain required to overcome the total loss of quantum dot laser has been decreased by $8 \%$ and $9.6 \%$ although the mirror loss has been increases by $5.23 \%$ and $9.52 \%$ using InN based quantum dot in the active layer of the laser structure with respect to that of using GaN and AlN respsctively. Furthermore, the turn-on delay of InN quantum dot based laser has been minimized significantly by $3.53 \%$ and $4.75 \%$ with respect to that of using GaN and AlN respsctively, which indicates the potentiality of InN based dot to fabricate high speed laser. The analytical results ascertain that $\mathrm{InN}$ can be a promising material for fabricating quantum dot based laser for high speed data transmission with the improved dependence of threshold current on the losses and gain phenomena of the laser.

\section{REFERENCES}

[1] K. L. Wang, D. Cha, J. Liu, C. Chen. Ge/Si. "self-assembled quantum dots and their optoelectronic device applications" Proceedings of the IEEE, 2007; 95(9):1866-1883. 2007

[2] L. Lester, The Laser Trailblazer, Innovative Research. The University of New Mexico, School of Engineering, 2008.

[3] D. Bimberg, M. Grundmann, F. Heinrichsdorff, N. N. Ledentsov, V. M. Ustinov, A. E. Zhukov, A. R. Kovsh, M. V. Maximov, Y. M. Shernyakov, B. V. Volovik, A. F. Tsatsul'nikov, Quantum dot lasers: breakthrough in optoelectronics, Thin Solid Films. 2000; 367(1): 235-249.

[4] Y. Arakawa, T. Someya, K. Tachibana,"Growth and physics of nitride-based quantum dots for optoelectronics applications, "Int. workshop on nitride semiconductor, IPAP conference Series, 1: 403-408.

[5] Y. Arakawa, H. Sakaki, "Multidimensional quantum well laser and temperature dependence of its threshold current," AppliedPhysics Letters, 1982; 40(11):939-941.

[6] S. A. Gaikwad, E. P. Samuel, D. S. Patil, D. K. Gautam, "Theoretical analysis of effect of temperature on threshold parameters and field intensity in GaN material based heterostructure," Bulletin of Materials Science. 2007; 30(3): 255261.

[7] S. H. Wei, "III-Nitride Semiconductors for Photovoltaic Applications,“" National Renewable Energy Laboratory (NREL), Golden, CO.In National center for Photovoltaics and Solar Program Review Meeting, Denver, Colorado, USA, 2003, (No. NREL/CP-590-33560).

[8] M. A. Humayun, M. A. Rashid, F. A. Malek, A. N. Hussain, "Effect of latticeconstant on band-gap energy and optimization and stabilization of high-temperature $\operatorname{In}_{\mathrm{x}} \mathrm{Ga}_{1-\mathrm{x}} \mathrm{N}$ quantum-dot lasers, “ Journal of Russian Laser Research. 2012, 33(4): 387-394.

[9] M. A. Humayun, M. A. Rashid, F. A. Malek, A. N. Hussain, I. Daut, "Design of quantum dot based laser with ultralow threshold current density,“ Applied Mechanics and Materials, 2012; 229(1639-1642).

[10] J. Houlihan, G. Huyet, J. G. McInerney, "Dynamics of a semiconductor laser with incoherent optical feedback," Optics communications. 2001; 199(1):175-179.

[11] M. A. Humayun, S. Khan, A. H. M. Alam, M. F. Abdul Malek, M. A. Rashid, "Reduction of cavity length dependence and improvement of characteristics of $1.55 \mu \mathrm{m}$ quantum dot based LASER using indium nitride, "Journal of Optoelectronics and Advanced Materials. 2017, 19(5-6): 298-302.

[12] L. V. Asryan, R. A. Suris, "Theory of Threshold Characteristics of Quantum Dot Lasers," Russian Academy Of Sciences Saint Petersburg Ioffe Physical-Technical Institution : 02I.

[13] G. P. Agrawal, G. R. Gray, "Effect of phase-conjugate feedback on the noise characteristics of semiconductor lasers," Physical Review. 1992; 46(9): 5890 
[14] O. Qasaimeh, "Effect of inhomogeneous line broadening on gain and differential gain of quantum dot lasers," IEEE Transactions on Electron Devices, 2003; 50(7): 1575-1581.

[15] M.M. Hossain, M. A. Humayun, M. Hasan, M. T. Bhuiyan, A. G. Hashimoto, A. Yamamoto, "Proposal of high performance $1.55 \mu \mathrm{m}$ quantum dot heterostructure laser using InN,“ IEICE Transactions on Electronics. 2012; 95(2): 255-261.

[16] I. Vurgaftman, J.R. Meyer, "Effects of bandgap, lifetime, and other nonuniformities on diode laser thresholds and slope efficiencies," IEEE Journal of Selected Topics in Quantum Electronics. 1997; 3(2): 475-484.

[17] L. V. Asryan, R. A. Suris, "Theory of threshold characteristics of quantum dot lasers: Effect of quantum dot parameter dispersion," International journal of high speed electronics and systems, 2002; 12(1): 111-176

[18] A. E. Zhukov, A. R. Kovsh, V. M. Ustinov, Z. I. Alferov, "Loss multiplication in a quantum dot laser," LASER PHYSICS-LAWRENCE, 2003; 13(3): 319-323

[19] C. Wang, F. Grillot, J. Even, "Impacts of wetting layer and excited state on the modulation response of quantum-dot lasers,“ IEEE Journal of Quantum Electronics. 2012; 48(9): 1144-1150. 